\title{
Basal cuspal tear of a porcine bioprosthetic mitral valve causing massive mitral regurgitation
}

\author{
Muhammed Tamim ${ }^{1}$, CHRISTOS ALEXIOU ${ }^{1}$, Yaser AlKady ${ }^{1}$, Mohsen Mahmoud ${ }^{1}$, and \\ Fatima Qaddoura ${ }^{1}$ \\ ${ }^{1}$ King Fahd Military Medical Complex
}

May 8, 2020

\begin{abstract}
Although porcine mitral bioprostheses provide predictably good long-term outcomes, unexpected leaflet tears leading to abrupt haemodynamic changes may occur. We report on a patient who presented with acute dyspnea due to cuspal tear of a porcine bioprosthetic mitral valve causing severe mitral regurgitation, her condition was subsequently complicated by systemic infection, probably pneumonia, and was successfully managed with urgent redo-mitral valve replacement.
\end{abstract}

\section{Key Words}

Cardiovascular pathology

Valve repair/replacement

\section{INTRODUCTION}

Appropriate follow-up after surgical implantation of bioprostheses ensures the timely detection of dysfunctional heart valves and their replacement in a safe manner $(1,2)$. Nonetheless, catastrophic structural valve deterioration can occur (1-5) early or late postoperatively (6.7). We describe the clinical presentation, management and outcome of a patient who suffered cuspal tear of a bioprosthetic mitral valve four years post valve

implantation, and discuss her case in context with the pre-existing relevant literature.

\section{CASE HISTORY}

A 53-year old female patient was admitted in a peripheral hospital with severe, acute onset dyspnea and orthopnea. Her past medical history included bioprosthetic aortic valve replacement (AVR) and mitral valve replacement (MVR) 4 years ago. Two hours later, she required intubation and mechanical ventilation, and transthoracic ECHO showed significant MR. Two days later, she developed high grade fever (39C), accompanied by radiological changes compatible with right lower lobe pneumonia, and a sharp rise in serum inflammatory markers. Multiple blood cultures, skin and nasopharyngeal swabs were taken, and she was commenced on i.v. antibiotics (Tamiflu, Tazocin, Azithromycin). All cultures returned negative including those for MERS-COV and H1N1. Meanwhile, she grewMethicillin Resistant Staphylococcus Aureus in a nasopharyngeal swab and the antibiotic treatment was changed to i.v. Meropenem, Vancomycin and Levofloxacin. Transesophageal echo (TEE) showed severe MR and malfunction of the bioprosthetic MV and was referred to our center.

On admission to the intensive care unit, she was mechanically ventilated, highly pyrexial, on high doses of noradrenaline. Repeat TTE showed a flail mitral valve leaflet and severe MR (Figures 1,2, 2D TEE), (Figures 
3, 3D TEE), dilated left atrium (LA), elevated systolic pulmonary artery pressure ( $75 \mathrm{mmHg})$, moderate tricuspid regurgitation (TR), accelerated flow across the aortic bioprosthesis (mean gradient $32 \mathrm{mmHg}$ ) and preserved bi-ventricular function. Computed tomography of the chest and abdomen showed right lower lobe consolidation consistent with pneumonia. She continued to run high fever and to require high doses of vasopressors and inotropes in order to maintain adequate blood pressure and urine output. Considering persisting fever, the unsatisfactory response to antibiotic therapy, severe haemodynamic compromise caused by the mechanical dysfunction of the prosthetic MV and the risk for irreversible multiorgan failure, a decision was taken to proceed with urgent surgical intervention seven days after her admission to our hospital.

A standard redo-MVR and De Vega TV annuloplasty were performed through a median re-sternotomy, utilizing cardiopulmonary bypass (aortic and bicaval cannulation), antegrade delivery of Del Nido cardioplegia and a right atrial-transeptal route to access LA and the MV. On inspection, one of the leaflets of the prosthetic MV was detached across its base from the frame of the valve, almost from commissure to commissure (Figure 4). The valve otherwise appeared normal without vegetations, thrombus or signs of valve dehiscence. The bioprosthesis was excised and after thorough tissue debridement and washing with normal saline, a mechanical valve (27mm On-XCryoLife, Inc. NW, U.S.A) was implanted. The LA appendage was obliterated with continuous suture, the interatrial septum closed, and the operation completed in the standard manner. Intraoperative TEE showed moderate biventricular dysfunction, well seating and normally functioning prosthetic MV without paravalvular leak, and mild TR.

Six days after surgery, the inotropes were stopped, the patient became afebrile and was extubated. Thereafter, she made steady progress becoming fully ambulant; Warfarin was commenced aiming for an INR of 2.5-3. Culture of the explanted valve was negative as were the tissues and fluids taken at operation. The patient was eventually transferred to the referring hospital three weeks after her operation for convalescence and completion of her antibiotic therapy. Pre-discharge ECHO showed well-functioning valves, mild TR, and mildly impaired bi-ventricular function.

\section{DISCUSSION}

Bioprosthetic valves have good durability and a predictable mode of failure, with gradual degeneration of the leaflet tissue being the norm (1-3). As a result, when a re-operation is required, is usually done in a controlled and safe manner (1-3). Nevertheless, some patients do experience sudden valve dysfunction due to acute structural valve deterioration, needing urgent attention $(1,2,3,6,7)$.

In a cohort of 836 patients who had a porcine bioprosthetic valve replacement, 28 required a re-operation with 18 of them having a leaflet tear of the explanted valve. Of these 18 patients, 12 had a previous MVR and 6 had AVR (3). Thus, the proportion of bioprosthetic valve leaflet tear leading to a re-operation was 2.1.\% (3). Cusp rupture or detachment in a mitral bioprosthesis may cause massive MR with serious consequences (3-7). Amongst the 12 patients who had a leaflet tear in a mitral bioprosthesis in Pomar's series, 6 presented in NYHA III and 6 in NYHA IV (3).

The initial diagnosis in our patient was cardiogenic pulmonary oedema, which suggests that the cuspal tear of the bioprosthesis and the ensuing massive MR was

the primary event. Subsequent clinical, radiological, laboratory and echocardiographical findings made right lobar pneumonia and/or infective endocarditis (IE) a likely diagnosis, even though serial blood cultures were negative. It is well known that approximately $1 / 3$ of patients with IE have sterile cultures (8). In either case, considering the major hemodynamic burden caused by the massive MR, urgent surgical intervention was in our patient imperative (8). Notably, all 6 patients who had cuspal tear of a bioprosthetic MV went on to have redo MVR in the elegant study by Pomar (3).

Single or multiple cuspal tears, leaflet perforations, free-edge tears and basal tears have all been described with the later (also seen in our patient) being the commonest (3-7). Some torn leaflets are calcified whereas in others calcification is visible only under light microscopy (3). In our patient, leaflet tear occurred at the base across its attachment onto the frame of the prosthetic valve (Figure 4) bearing no calcium, vegetations 
or other sign suggestive of infection, and valve culture was negative. These implies that the detachment of leaflet from the frame of the valve was a purely mechanical event (9).

Leaflet detachment occurred 4 years after surgery in our patient, which is in keeping with an average time for cuspal tears of around 4-6 years quoted by Pomar (3). However, following implantation of a mitral bioprosthesis, Miura (6) reported a cusp detachment occurring only 10 months after surgery and Ha et al (7) 12 years after surgery.

\section{CONCLUSIONS}

Although porcine MV bioprostheses provide predictably good long-term outcomes, unexpected cusp tears leading to abrupt haemodynamic changes with disastrous consequences may occur. This reminds us of the need for meticulous clinical and echocardiographical follow-up of these patients.

\section{REFERENCES}

1. Malvindi P, Mastro F, Kowalewski M et al. Durability of Mitral Valve Bioprostheses: A Meta-Analysis of Long-Term Follow-up Studies. Ann Thorac Surg. 2020;109(2):603-611.

2. Grunkemeier G, Furnary A, Wu Y, Wang L, Starr A. Durability of pericardial versus porcine bioprosthetic heart valves. J Thorac Cardiovasc Surg. 2010;144(6):1381-6.

3. Pomar L, Bosch X, Chaitman B, Pelletier C, Grondin C. Late Tears in Leaflets of Porcine Bioprostheses in Adults. Ann Thorac Surg 1983;37(1):78-83.

4. Gao, G., Wu, Y., Grunkemeier, G.L., Furnary, A.P., and Starr, A. Durability of pericardial versus porcine aortic valves. J Am Coll Cardiol . 2004; 44: 384-388.

5. Cipriano P, Billingham M, Oyer P, et al: Calcification of porcine prosthetic heart valves: a radiographic and light microscopic study. Circulation 66:1100, 1982.

6. Miura T, Hazama S, Iwasaki K, Izumi K, Matsukuma S, Eishi K. A Rapid Structural Degeneration of a Porcine Mitral Valve. Ann Thorac Surg 2012;93:e113-4.

7. Ha JW, Chang BC, Chung N, Cho SH. Images in cardiology. Acute severe mitral regurgitation due to unusual detachment of bioprosthetic valve leaflet. Clin Cardiol . 2000;23(3):213.

8. Alexiou C, Langley SM, Stafford H, Lowes AJ, Livesey SA, Monro JL. Surgery for active culturepositive endocarditis: Determinants of early and late outcome. The Annals of Thoracic Surgery 2000;69:1448-1454

9. Koziarz A, Makhdoum A, Butany J, Ouzounian M, Chung J. Modes of bioprosthetic valve failure: a narrative review. Current Opinion in Cardiology. 2020 Mar;35(2):123-132.

\section{Figure Legends}

Figure 1. Two-dimensional transesophageal ECHO showing flail leaflet of the bioprosthetic mitral valve.

Figure 2. Doppler 2D transesophageal ECHO showing a large mitral regurgitant jet.

Figure 3. Three-dimensional color Doppler transesophageal ECHO showing a large mitral regurgitant get.

Figure 4. Explanted mitral valve bioprosthesis basal cusp tear (blue arrow) almost from commissure to commissure.

\section{Hosted file}

Figure 1..docx available at https://authorea.com/users/312821/articles/449312-basal-cuspaltear-of-a-porcine-bioprosthetic-mitral-valve-causing-massive-mitral-regurgitation

\section{Hosted file}

Figure 2.docx available at https://authorea.com/users/312821/articles/449312-basal-cuspaltear-of-a-porcine-bioprosthetic-mitral-valve-causing-massive-mitral-regurgitation

\section{Hosted file}


Figure 3.docx available at https://authorea.com/users/312821/articles/449312-basal-cuspaltear-of-a-porcine-bioprosthetic-mitral-valve-causing-massive-mitral-regurgitation

\section{Hosted file}

Figure 4.docx available at https://authorea.com/users/312821/articles/449312-basal-cuspaltear-of-a-porcine-bioprosthetic-mitral-valve-causing-massive-mitral-regurgitation 